\title{
Hypersonic Magneto-Fluid-Dynamic Compression in Cylindrical Inlet
}

\author{
J.S. Shang and C.L. Chang* \\ Department of Mechanics and Materials Engineering \\ Wright State University \\ Dayton, $\mathrm{OH}$ \\ joseph.shang@wright.edu \\ Computational Aeroscience Branch* \\ NASA Langley Research Center \\ Hampton, VA
}

\begin{abstract}
Hypersonic magneto-fluid-dynamic interaction has been successfully performed as a virtual leading-edge strake and a virtual cowl of a cylindrical inlet. In a side-by-side experimental and computational study, the magnitude of the induced compression was found to be depended on configuration and electrode placement. To better understand the interacting phenomenon the present investigation is focused on a direct current discharge at the leading edge of a cylindrical inlet for which validating experimental data is available. The present computational result is obtained by solving the magneto-fluid-dynamics equations at the low magnetic Reynolds number limit and using a nonequilibrium weakly ionized gas model based on the drift-diffusion theory. The numerical simulation provides a detailed description of the intriguing physics. After validation with experimental measurements, the computed results further quantify the effectiveness of a magnet-fluid-dynamic compression for a hypersonic cylindrical inlet. At a minuscule power input to a direct current surface discharge of 8.14 watts per square centimeter of electrode area produces an additional compression of 6.7 percent for a constant cross-section cylindrical inlet.
\end{abstract}

\section{Nomenclature}

$\begin{array}{ll}\mathbf{B} & \text { Magnetic flux density } \\ \mathbf{F} & \text { Flux vector of the MFD equations } \\ \mathrm{n}_{\mathrm{e}}, \mathrm{n}_{+} & \text {Number density of charged particles } \\ \mathbf{q} & \text { Dependent variable vector } \\ \mathbf{u} & \text { Velocity vector } \\ \beta & \text { Recombination coefficient } \\ (\xi, \eta, \zeta) & \text { Transformed coordinate } \\ \sigma & \text { Electric conductivity } \\ \tau & \text { Shear stress tensor }\end{array}$

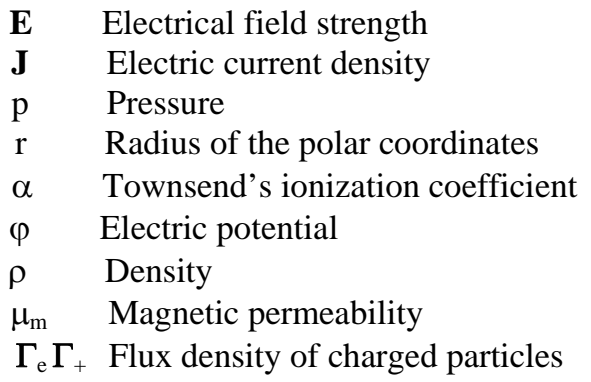

\section{Introduction}

At present, the scramjet appears to be the most promising hypersonic propulsion system for its simplicity in construction and relatively few components in comparison with other systems [1,2]. The propulsion requirement for high-speed flight varies greatly from take-off to cruising condition and cannot be efficiently supported by a fixed configuration inlet. To improve propulsive efficiency in an operation range, modification of the inlet may be the most cost effective. However once a variable configuration inlet is implemented by an array of compression ramps and boundary-layer control slots, a complicated mechanical flow control must be adopted that leads to an avoidable weight penalty. Meanwhile when operating beyond the design condition, managing and eliminating parasitic effects increases the complexity of the propulsive system. For this reason an alternative, non-intrusive, rapid response, flow control mechanism, other than mechanical means, is very appealing.

Numerous ideas have been advocated for using electromagnetic force for high-speed flow control [3-14]. Some of the research efforts include an externally applied magnetic field in an attempt to accentuate the magneto-fluiddynamic (MFD) interaction by invoking the Lorentz force in addition to Joule heating [3-9,12,13]. Surzhikov and Shang [14] have shown that the Hall current exerts significant influence to the plasma generation via the electron collision process. The Hall current can even suppress the MFD interaction when the value of the Hall parameter 
attains an exceedingly high value. However, in a relatively weak applied magnetic field, B $\leq 0.2$ Tesla, the interaction is enhanced by the presence of an externally applied magnetic field. In a numerical simulation around a cone in hypersonic flow, Borghi et al [13] also found that the Hall current can significantly weaken the MFD interaction. All the aforementioned computing simulations are in a general agreement with experimental observations by Bityurin et al [7] on the effect of Hall current in MFD interactions.

The inefficient plasma generation process has prevented the plasma actuator from becoming a cost effective device for flow control or aerodynamic performance enhancement [15-17]. Shang et al [9,18,19] conducted a series of side-by-side computational and experimental investigations to show that a small electromagnetic perturbation near the hypersonic leading edge can be amplified by the viscous-inviscid interaction to become an effective flow control mechanism. They first demonstrated that the MFD interaction can perform as a virtual leading edge strake. Using a power supply of 50 Watts to the surface plasma discharge at Mach five, the MFD interaction induces a compression over an immobile surface that acts as though this control surface has executed a one-degree pitching movement $[8,9]$. More than a five-degree equivalent pitching angle has been produced by using a total power supply of 350 Watts to the plasma actuator. The similar idea has also been applied successfully to a rectangular constant crosssection area inlet to perform as a virtual inlet cowl [18,19].

The basic operating principle of the dielectric barrier discharge (DBD) high-speed flow control mechanism is a combination of a small electromagnetic perturbation and a subsequent amplification by the viscous-inviscid interaction. A simple direct current discharge (DCD) near the sharp leading edge of a configuration introduces three mechanisms for flow control; the volumetric Joule heating, convective electrode heating, and the electrostatic force. The dissipative Joule heating is the consequence of electric current movement in a partially ionized gas, the electric conductivity of this medium is typically lower than one mho/m [19]. The magnitude of the Joule heating associated with the DCD is around $10 \%$ of the power required for the surface plasma generation [8,9,14]. For most applications, it has a range up to 10 watts $[18,19]$. The electron collision process for plasma generation also results in an electrode temperature around $500 \mathrm{~K}[8,9]$. In the testing environment of a plasma channel, this electrode temperature is much higher than the model surface temperature and results in a convective heat transfer to the air stream. Based on the tested electrode surface area, the total amount of convective energy transfer is 6.6 watts. Meanwhile an electrostatic force exists adjacent to the electrodes where the space discharge separation occurs in the plasma sheath, the orientation of this force is dictated by the local electric field. The magnitude of the electrostatic force is around $1 \mathrm{kN} / \mathrm{m}^{3}$, much less than that of dielectric barrier discharge, and is deeply imbedded in the cathode layer $[14,20]$. For this reason, all these electromagnetic perturbations exist only in the inner portion of a boundary layer. However, the energy transfer is dominant over that of the momentum transfer for DCD flow control.

To determine the application range of MFD for flow control, the present approach will demonstrate that this flow control mechanism can be equally applicable to a cylindrical inlet. The most obvious choice of the electrode placement is at the sharp leading edge where the flow is laminar and the intensity of the perturbation can be relatively weak, but exerts profound influence on the entire flow field. The classic hypersonic flow theory by Hayes and Probstein describes a inviscid-viscous interaction over a sharp leading edge as the pressure interaction [21]. The induced pressure distribution near the leading edge of a solid surface can substantially alter the growth rate of the displacement thickness of the boundary layer to form a closed feedback loop. The outward deflection of the streamlines and the resulting high-pressure region can reach far downstream. The magnitude of the induced pressure is well-known and can be calculated by a single interaction parameter $\chi=M^{3}(\mathrm{C} / \text { Rey })^{1 / 2}$. The viscousinviscid interaction is strongly amplified by hypersonic flows due to its dependence on the cube power of the Mach number. This control mechanism can be repetitiously activated in microseconds. Results from both experimental and computational efforts have shown the chain of events constitute a very effective hypersonic flow control technique $[18,19]$.

The classic MFD equations at the low magnetic Reynolds limits are solved to duplicate the experimental observation in a cylindrical inlet model [20]. The partially ionized air models are utilized ranging from a simple phenomenological approximation to the rigorous drift-diffusion theory to describe the nonequilibrium direct current discharge (DCD) [21]. The axisymmetric numerical results are first calibrated with the measured pitot pressure surveys, and then evaluated according to the critical aerodynamic parameters for inlet performance. The temperature and density contours, Pitot and static as well as stagnation pressure distributions are presented to describe the overall flow field structure of the MFD compression. 


\section{Governing Equations}

In most aerodynamic applications of flow control using a plasma actuator, the Magnetic Reynolds number is much less than unity, $\mathrm{Re}_{\mathrm{m}}=\mathrm{u} \sigma \mu_{\mathrm{m}} \mathrm{L}<<1$ [16,17]. According to the investigated flow condition, the $\mathrm{Re}_{\mathrm{m}}$ has a value of $8.48 \times 10^{-6}$, thus the governing equations of the low Magnetic Reynolds number approximation are fully justified for the present investigation [16]:

$$
\begin{aligned}
& \partial \rho / \partial t+\nabla \cdot(\rho \mathbf{u})=0 \\
& \partial \rho \mathbf{u} / \partial \mathrm{t}+\nabla \cdot(\rho \mathbf{u u}-\tau)=\mathbf{J} \times \mathbf{B} \\
& \partial \rho \mathrm{e} / \partial \mathrm{t}+\nabla \cdot(\rho \mathbf{u}-\mathbf{q}-\mathbf{u} \cdot \tau)=\mathbf{E} \cdot \mathbf{J}
\end{aligned}
$$

The electrostatic force term, $\rho_{\mathrm{e}} \cdot \mathbf{E}$, is omitted by the traditional formulation [16,17]. This simplification in the present analysis is also based on the fact that the electrostatic force is around 43 dyne $/ \mathrm{cm}^{3}$, or $430 \mathrm{~N} / \mathrm{m}^{3}$, which exists within the thin plasma sheath mostly over the cathode and exerts downward. It is negligible in comparison with the inertia of the oncoming hypersonic flow. Again in the absence of an applied external magnetic field to the DCD, the Lorentz force, $\mathbf{J} \times \mathbf{B}$ in equation (1-2) is also relatively insignificant, but is retained for a possible externally applied magnetic field.

The governing partial differential equation system is identical to the Navier-Stokes equations except the non-zero source terms. The DCD has the maximum charged particle number density over the cathode and has a maximum value around $8.8 \times 10^{11} / \mathrm{cc}$, the electrical conductivity is less than 1 mho per meter locally. At the experimental stagnation pressure of 580 Torr $(76.3 \mathrm{Kpa}$ ) and Mach number of 5.15 , the air number density in the test section is $1.57 \times 10^{17} / \mathrm{cc}$; thus the mass fraction of the charged particles is $10^{-5}$ to $10^{-6}$. It is therefore justifiable to consider only the transport properties of the weakly ionized air and to neglect the effects of the nonequilibrium thermodynamics and chemical kinetics.

For the MFD compression, the electromagnetic perturbation enters the interaction mostly as the volumetric Joule heating and convective electrode heating. These two fundamentally heat transfer processes occur at vastly different time scales; the former takes place at the instant when the plasma is ignited on the order of microseconds, and the heat release is confined to within a few Debye lengths immediately above the electrodes. The convective heat transfer on the other hand will not reach an equilibrium state until a few minutes later [8,9]. The heating effects thicken the displacement thickness of the shear layer on the inlet surface, however it is additive. To model this perturbation, a wide range of formulations of the electromagnetic perturbation is possible, but the total amount of energy released to the air stream is calculated from the direct current gas discharge based on the drift-diffusion theory. Surzhikov and Shang [20] have successfully developed a model of a three-component plasma (neutral, electron, and ion) and two-temperature plasma:

$$
\begin{aligned}
& \partial \mathrm{n}_{\mathrm{e}} \partial \mathrm{t}+\nabla \cdot \Gamma_{\mathrm{e}}=\alpha(\mathrm{E}, \mathrm{p})\left|\Gamma_{\mathrm{e}}\right|-\beta \mathrm{n}_{+} \mathrm{n}_{\mathrm{e}} \\
& \partial \mathrm{n}_{+} / \partial \mathrm{t}+\nabla \cdot \Gamma_{+}=\alpha(\mathrm{E}, \mathrm{p})\left|\Gamma_{\mathrm{e}}\right|-\beta \mathrm{n}_{+} \mathrm{n}_{\mathrm{e}} \\
& \Gamma_{\mathrm{e}}=-\mathrm{D}_{\mathrm{e}} \nabla \mathrm{n}_{\mathrm{e}}-\mathrm{n}_{\mathrm{e}} \mu_{\mathrm{e}} \mathbf{E} \\
& \Gamma_{+}=-\mathrm{D}_{+} \nabla \mathrm{n}_{+}+\mathrm{n}_{+} \mu_{+} \mathbf{E}
\end{aligned}
$$

In the above formulation, $\alpha(\mathrm{E}, \mathrm{p})$ and $\beta$ are the first Townsend ionization coefficient and recombination coefficient. The parameters $\mu_{\mathrm{e}}$ and $\mu_{+}$are the electron and ion mobility, and $\mathrm{D}_{\mathrm{e}}$ and $\mathrm{D}_{+}$are the electron and ion diffusion coefficients [15,21]. The electrical current density appears in the low Magnetic Reynolds number approximation as:

$$
\mathbf{J}=\mathrm{e}\left(\Gamma_{+}-\Gamma_{\mathrm{e}}\right)
$$

A compatible electrical field intensity, $\mathbf{E}$, of the discharge domain is obtained by satisfying the charge conservation equation $[14,18]$. This equation is further simplified in a globally neutral plasma by introducing an electrical potential function, $\mathbf{E}=-\nabla \phi$. The electrical field intensity is then the solution of the well-known Poisson equation of plasmadynamics associated with the net space charge density, $\rho_{\mathrm{e}}$.

$$
\nabla^{2} \phi=-\rho_{\mathrm{e}} / \varepsilon
$$


The initial values and boundary conditions, as well as, the numerical procedure are directly usable from the cumulative knowledge from the CFD discipline [8-12]. For the velocity components, the free-stream and the nochange condition are prescribed at the entrance, far field, and exit boundaries of the computational domain respectively. The no-slip condition applies to all the velocity components on the inlet solid surface. The adiabatic condition is used to determine the gas temperature on the inlet surface, except that of the electrodes. Finally, the surface pressure is evaluated by the vanishing normal pressure gradient condition locally.

The initial values and boundary conditions of the plasma model for a numerically stable procedure have been found through a series of sustained research efforts [8,9,10,14,21]. A key element in determining the boundary conditions is specifying the electron number density on the cathode for the secondary emission phenomenon. This physical requirement is met by specifying that the normal component of the electron flux at the cathode be proportional to its ion counterpart [20].

$$
\Gamma_{\mathrm{e}} \cdot \mathrm{n}=-\gamma \Gamma_{+} \cdot \mathrm{n}
$$

All numerical results are obtained by solving the time-dependent conservation laws in mass-averaged variables. The spatial discretization involves a semi-discrete finite-volume scheme [23,24]. The upwind-biasing approximation is applied to the convective and pressure terms and central differencing is used for the shear stress and heat transfer terms.

\section{Numerical Procedure}

In the flux-difference splitting procedure for shock capturing, the flux vectors at the control surface are written as the solution to the approximate Riemann problem.

$$
\begin{aligned}
\delta \mathbf{F i} & \left.=1 / 2\left[\mathbf{F}\left(\mathbf{Q}_{\mathrm{L}}\right)+\mathbf{F}\left(\mathbf{Q}_{\mathrm{R}}\right)\right]-\left|\mathbf{M}_{\text {inv }}\right|\left(\mathbf{Q}_{\mathrm{R}}-\mathbf{Q}_{\mathrm{L}}\right)\right]_{\mathrm{i}+1 / 2} \\
& \left.-1 / 2\left[\mathbf{F}\left(\mathbf{Q}_{\mathrm{L}}\right)+\mathbf{F}\left(\mathbf{Q}_{\mathrm{R}}\right)\right]-\left|\mathbf{M}_{\text {inv }}\right|\left(\mathbf{Q}_{\mathrm{R}}-\mathbf{Q}_{\mathrm{L}}\right)\right]_{\mathrm{i}+1 / 2}
\end{aligned}
$$

where $\mathbf{Q}_{\mathrm{L}}$ and $\mathbf{Q}_{\mathrm{R}}$ are interpolated values of the dependent variables, $\rho$, $\rho \mathrm{u}, \rho \mathrm{v}, \rho \mathrm{w}$, and $\rho \mathrm{e}$ at the interface of the control volume and $\mathrm{M}_{\mathrm{inv}}$ is the Jacobian matrix of the inviscid or convective terms [23].

A slope limiter is also used to control the discontinuous pressure jumps at the shock front. Time advancement is implicit to solve that the flows have a steady state asymptote. A min-mod limiter is adopted for the present computations.

$$
\begin{aligned}
& \left(\mathbf{Q}_{\mathrm{L}}\right)_{\mathrm{i}+1 / 2}=\mathbf{Q}_{\mathrm{i}}+1 / 4[(1-\kappa) \nabla+(1+\kappa) \Delta]_{\mathrm{i}} \\
& \left(\mathbf{Q}_{\mathrm{R}}\right)_{\mathrm{i}+1 / 2}=\mathbf{Q}_{\mathrm{i}+1}-1 / 4[(1-\kappa) \Delta+(1+\kappa) \nabla]_{\mathrm{i}+1}
\end{aligned}
$$

The min-mod operators are defined as;

$$
\begin{aligned}
\nabla & =\operatorname{minmod}[\nabla,(3-\kappa) /(1-\kappa) \Delta] \\
\Delta & =\operatorname{minmod}[\Delta,(3-\kappa) /(1-\kappa) \nabla]
\end{aligned}
$$

For convergence acceleration, a three-level mesh sequencing of the multigrid technique is applied [24].

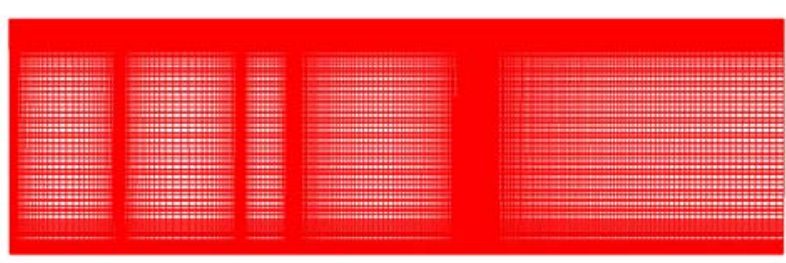

Figure 1. $(2 \times 93 \times 301)$ mesh system
For the axisymmetric configuration, only the upper half of the radial plane is included in the computational domain [20]. The numerical simulations are generated on three mesh systems, $(2 \times 81 \times 229),(2 \times 81 \times 301)$ and $(2 \times 93 \times 301)$ to better capture the invert conical shock originating from the leading edge of the model. The minimum grid spacing immediately adjacent to the sidewall is one hundredth of the laminar boundary thickness at the 
exit plane $\left(4.37 \times 10^{-2} \mathrm{~cm}\right)$. A compressed mesh is also implemented at the leading edge of the inlet with four streamwise cross-section planes to define the freestream. A high mesh density is also provided at the edges of electrodes and the anticipated apex of the conical shock for better numerical resolution. The finest mesh system of the present investigation is depicted in Figure 1.

In Figure 2, the computed density contours in the upper-half-plane of the flow field on three progressively refined mesh systems are presented. The coarsest mesh has only the high grid density in the inner domain of the boundary

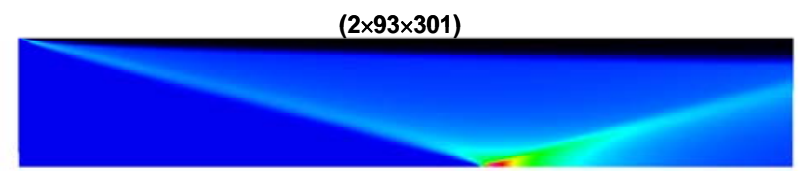

$(2 \times 81 \times 301)$

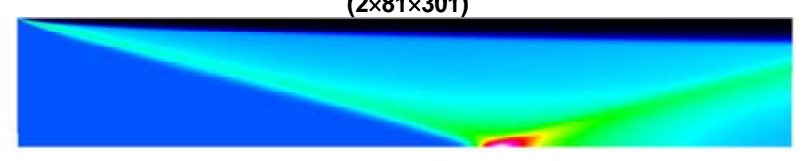

$(2 \times 81 \times 229)$

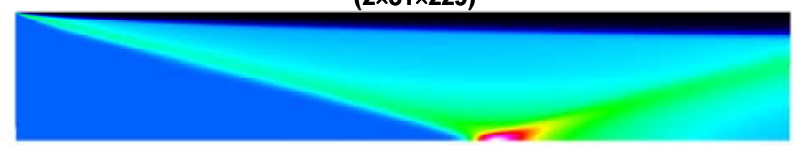

Figure 2. Comparison of density contours on three different grid systems

results is $0.8 \%$. layer, leading edges of the model, and the edges of electrodes $(2 \times 81 \times 229)$. The medium mesh system is enriched by an additional mesh clustering at the anticipated conical shock apex $(2 \times 81 \times 301)$. The finest mesh system includes a further mesh space refinement at the axis of symmetry $(2 \times 93 \times 301)$. For all mesh system used, the ratio between the finest axial and radial mesh spaces is a factor of ten $\left(\Delta x=4.37 \times 10^{-3}, \Delta r=4.37 \times 10^{-4} \mathrm{~cm}\right)$. The finest mesh spacing is located immediately adjacent and normal to the inlet sidewall. As clearly shown in Figure 2; all numerical simulations capture the essential feature of the bi-conical shock structure. The locations of the shock apex are better defined as the mesh density is enriched. The result from the refined mesh shows a progressive movement of the shock apex toward upstream, however the maximum deviation among all

\section{Flow-Field Structure}

The present numerical simulation duplicates the experimental effort to generate a virtual variable geometric inlet

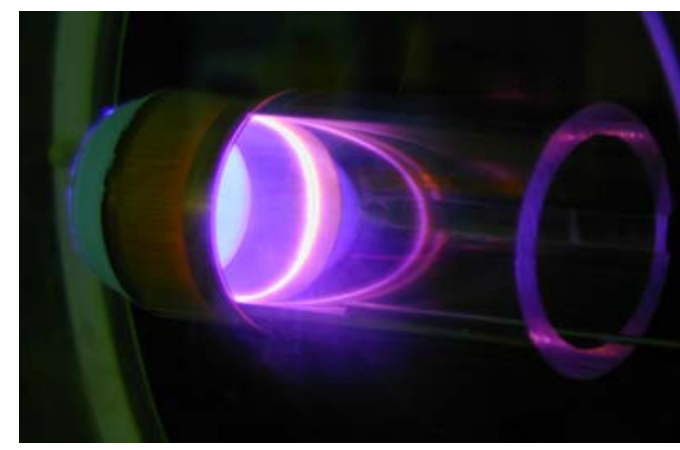

Figure 3. Activated DCD, $\varphi=480 \mathrm{~V}, \mathrm{I}=50 \mathrm{~mA}$ cowl by using the DCD within a constant cross-section cylindrical inlet model. The freestream Mach number has a nominal value of 5.15 and the stagnation temperature is 270 $\mathrm{K}$; thus the static temperature of the unperturbed freestream is $43 \mathrm{~K}$. To ensure a stable inflow condition for the channel with the cylindrical inlet model, tests are conducted at two stagnation pressures, 370 and 580 Torr. These conditions produce two Reynolds numbers based on model lengths of $1.66 \times 10^{5}$ and $2.52 \times 10^{5}$ respectively [24]. In the data reduction process, the most reliable data were found at the higher stagnation pressure condition; therefore the presented numerical simulation concentrates on this experimental condition.

For the constant cross-sectional area inlet model, the overall length of the model is $10.16 \mathrm{~cm}$ and outer and inner diameters are $4.44 \mathrm{~cm}$ and $3.49 \mathrm{~cm}$ respectively. The cathode and anode are embedded in the sidewall normal to the $\mathrm{x}$ coordinate. The cathode ring has a width of $1.43 \mathrm{~cm}$ and the width of the anode is $0.64 \mathrm{~cm}$. The separation

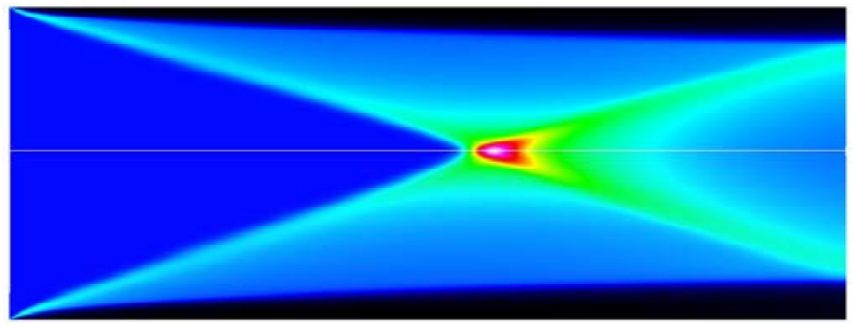

Figure 4, Density contour within the cylindrical inlet, $\mathrm{M}=5.15$, Rey $=2.52 \times 10^{5}$ distance between the electrodes is $1.59 \mathrm{~cm}$ and the weakly ionized air is sustained by an electric potential of $480 \mathrm{~V}$ for currents from 50 to $150 \mathrm{~mA}$. The cathode is placed at the leading edge of the model which has an outward bevel of 20 degrees; the nominally sharp leading edge actually has a small radius of curvature of $0.127 \mathrm{~mm}$. The actuated DCD in the cylindrical inlet is depicted in Figure 3. The DC discharge is viewed from the exit of the duct and at an oblique angle from the axis. 
The dominant visual feature of the discharge is the glow over the electrodes and the refection from the glass sidewall. Under this testing condition, the discharge current density on the anode is $6.78 \mathrm{~mA} / \mathrm{cm}^{2}$.

It is interesting to note that a trivially simple cylindrical inlet generates a rather intriguing shock wave structure within the hypersonic inlet. The viscous-inviscid interaction at the leading edge induces an inverted conical shock and the apex of the shock is located on the axis of symmetry. Downstream of the apex, the reflected shock wave continuously propagates towards the exit plane. In Figure 4, the computed density contours within the inlet are given; the numerical result is generated on the coarsest mesh system but still describes the basic shock wave structure. At the apex of the conical shock, the basic wave structure is uncertain. Since the stream is converging toward a single point and diverging immediately afterward, the existence of a Mach reflection is possible [25,26]. The Pitot pressure probe survey at the apex reveals a sharp spike, indicating a drastic shock structure adjustment. Unfortunately, this phenomenon occurs on the axis of symmetry where the numerical result must be generated on a line of a removable singularity. Instead of utilizing multiple numerical algorithms or a general 3D formulation, a mesh refinement approach is adopted in the present study.

Pressure profiles at different streamwise locations generated from the finest mesh systems are given in Figure 5. In order to better describe the static pressure patterns, the profiles upstream of the shock apex are

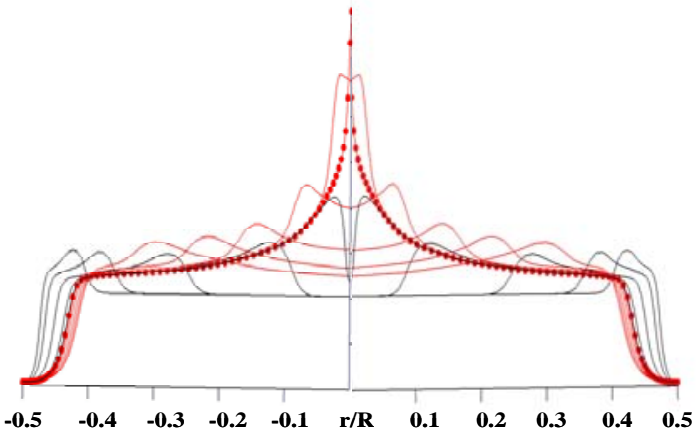

Figure 5. Static pressure profiles within the inlet model designated in black and the downstream variations are presented as the red traces. At the shock apex the pressure profile is presented in red connected discrete points. The numerical simulations capture a spike pressure behavior at the conical shock apex. Upstream of this point, the pressure profiles reveal a converging conical shock toward the apex. Immediately upstream of this location, the pressure distribution at the axis of symmetry exhibits a dip. Since there is no physical observation that can support this behavior, it is believe to a numerical artifact. Downstream of the shock apex, the numerical results indicate a divergent conical shock structure and increasing thickness of the wall shear layer. These computed results seem to indicate that the streamwise numerical resolution is sufficient to determine the location of the apex of the conical shock.

\section{Comparison with Experimental Data}

The direct current discharge is introduced into the internal flow field of a constant cross-sectional area cylindrical

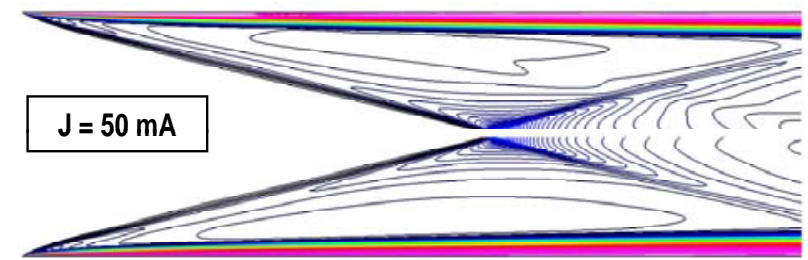

Figure 6. Simulated temperature field with/without DCD, $\varphi=402$ v J=50 mA, Rey=1.66 $\times 10^{5}$

at the lower Reynolds number condition, Rey $=1.66 \times 10^{5}$. model as a small perturbation. The interaction between the electromagnetic effect and the fluid dynamics is linked through the outward flow deflection by the thickened displacement thickness. In supersonic and hypersonic flow, the rate of change by the displacement thickness becomes the key parameter for the induced oblique shock in the viscous-inviscid interaction. The rate of change in displacement thickness directly corresponds to the power input for plasma generation. This observation can be easily made in Figures 6 and 7

In Figure 6, the composite temperature distributions for the inlet with and without an actuated DCD at an electric potential of 402 volts and circuit current of $50 \mathrm{~mA}$ are given. The simulated computation duplicates the experimental condition including the elevated electrode surface temperatures. According to the best estimate, the surface temperature of the cathode is $500 \mathrm{~K}$ and the anode has a lower value of $350 \mathrm{~K}[9,19]$. In this graph, the actuated DCD result is depicted in the upper half plane and the unperturbed flow in the lower half plane. As it was anticipated, at the low plasma generation power the Joule heating is determined to be less than 2 watts and the induced magneto-fluid dynamic compression is relatively small. This observation can be easily made in this 
comparative presentation; the resultant oblique shock is slightly steepened and intercepts the axis of symmetry upstream of the unperturbed flow.

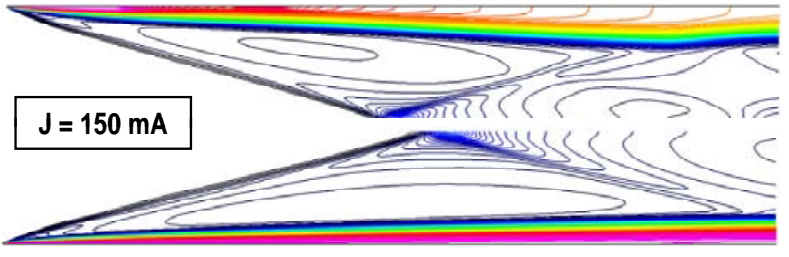

Figure 7. Simulated temperature field with/without DCD, $\varphi=460 \mathrm{v} \mathrm{J}=150 \mathrm{~mA}$, Rey $=1.66 \times 10^{5}$
However, the result is drastically different for the higher plasma power input in Figure 7 . The comparative study is generated by the actuator powered by 69.0 watts; the applied electric field potential of 460 volts and the circuit current of 150 $\mathrm{mA}$. An induced oblique shock now possesses a significant strength so that the oblique shock continuing after the shock focus impinges on the inner surface of the inlet model. The impinging shock

generates an unexpected and additional viscous-inviscid interaction near the exit plane of the inlet at the lower Reynolds number condition, Rey $=1.66 \times 10^{5}$. In the numerical simulation, the adverse pressure gradient even triggers incipient flow separation near the exit plane at the relatively low Reynolds number flow condition. Unfortunately, this result is not directly verifiable from the experimental observations [19].

This specific phenomenon can be further verified by the comparison of the streamwise Pitot pressure distributions along the axis of the inlet model in Figure 8. The computed and measured results of the actuated DCD are designated by the solid line and filled square symbols for the plasma actuated case and by the dash line and filled circles for the unperturbed condition. The computing simulations do not include the slightly blunt leading edge, thus have a weaker oblique shock. As a consequence, the shock focus is consistently formed downstream of the experimental observation and the divergent conical shock after passing through the shock focus does not impinge on the inlet side wall. The impinging shock of the experiments creates an adverse pressure gradient near the inlet exit plane which can induce either flow instability or incipient flow separation for the low Reynolds number flow. This behavior is different from the

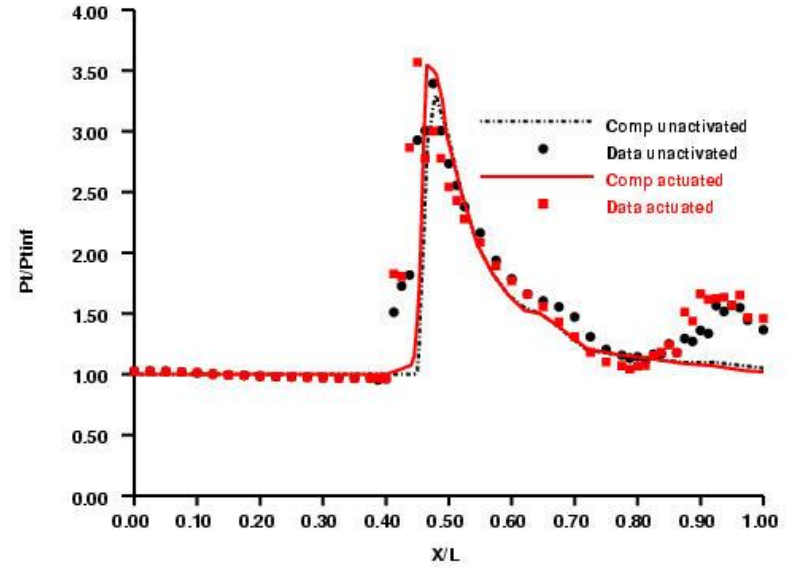

Figure 8. Comparison of Pitot pressure distributions along the axis of symmetry, Rey $=2.52 \times 10^{5}$

numerical simulations of the sharp leading edge cylindrical inlet in which the divergent shock exits the inlet uninterrupted.

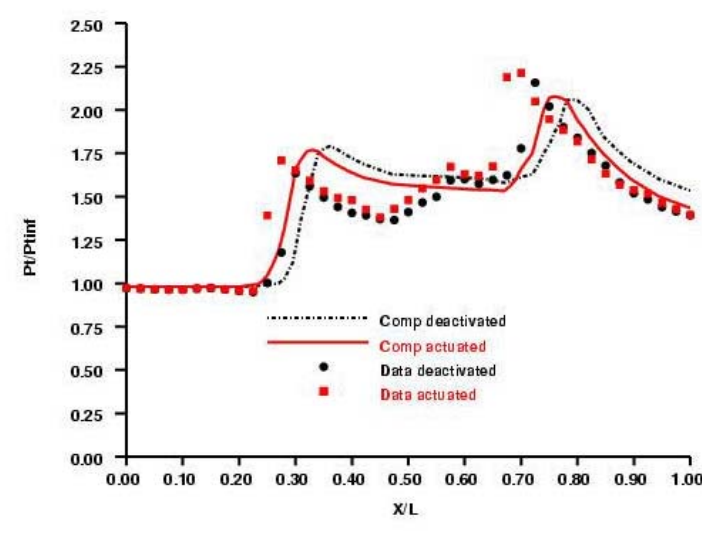

Figure 9. Comparison of off axial Pitot pressure distributions with/without DCD, Rey $=2.52 \times 10^{5}$
For the axisymmetic configuration, the characteristic thicknesses of the boundary layer are thinner in comparison with a two-dimensional counterpart. According to Mangler, the thickness is reduced by a factor of $\sqrt{3}$ on a conical configuration at the identical Reynolds number. The induced pressure is thus accordingly smaller and more difficult to resolve than the rectangular inlet. An added complication for the experimental effort is that the blockage of the inlet model for the blow-down free jet facility has approached its limit. A small fluctuating back pressure from the vacuum pumps amplifies the model blockage in the free jet. Careful back pressure was monitored during testing to maintain values of $8 \pm 0.5 \mathrm{~mm}$ HG, but a constant value was not sustainable. For this reason and the shock impingement interactions, the test data exhibit a wider data scatter near the exit which indicates the possibility of 
a local separated flow region and reflecting by the unsteady flow pattern [24]. In spite of the local discrepancy, both measured and computed results indicate a perceptible magneto-fluid-dynamic compression effect.

The comparison of Pitot pressure data and computing simulations in the axial direction, but at an off-axis location, $\mathrm{r} / \mathrm{R}=0.3$, is depicted in Figure 9. Although the data indicate a stronger shock strength than the computing simulation downstream of the conical shock focus, reasonably good agreement between experimental and computational results has been reached. In addition, these results further confirm the fact that the large data scattering band near the inlet exit is confined to a region close the wall of the inlet. More importantly, the data and computational results both clearly show a measurable magneto-fluid-dynamic compression produced by the simple DCD at the entrance region of the cylindrical inlet.

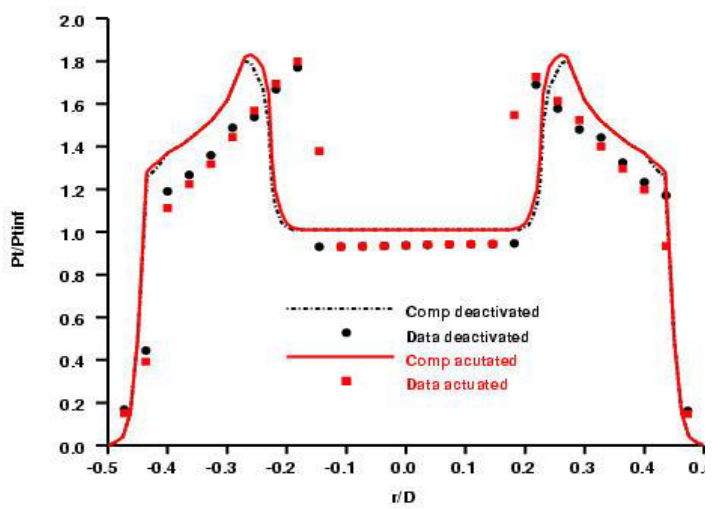

Figure 10. Pitot pressure profiles upstream of the conical shock focus, $\mathrm{X} / \mathrm{D}=1.3$, Rey $=2.52 \times 10^{5}$

Additional verifications of the numerical simulations is performed by comparing to experimental observations at a few cross-sectional planes of the inlet are also included. The Pitot pressure profiles at the axial location of $\mathrm{X} / \mathrm{D}=1.33$, upstream of the conical shock focus, is presented in Figure 10. It becomes obvious that the data is not symmetrical because of the misalignment of the model with respect to the centerline of the plasma channel. This pattern has also been detected from the experimental measurement of the horizontal and vertical Pitot pressure surveys [19]. In spite of this fact the computing simulations reproduce all the key features of the leading edge and DCD induced oblique shock. The agreement has been reached in the prediction of the shear layer thickness and the existence of an inviscid core. The overpredicted core size is completely consistent to the fact that the induced oblique shock from a slightly blunt leading edge possesses a greater shock angle and smaller inviscid core. At this upstream stream location, the difference between the unperturbed and actuated DCD flow fields is relatively small and is within the data scatter but is highlighted by the computed results.

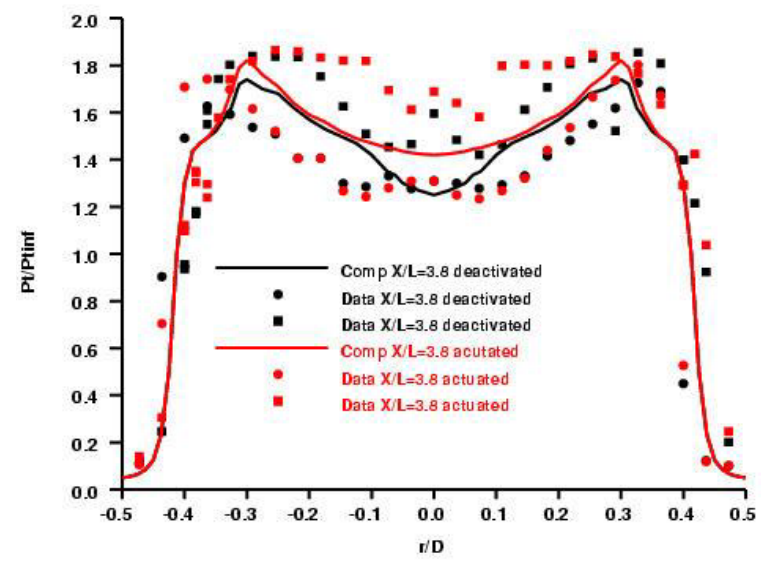

Figure 11. Pitot pressure profiles near inlet exit plane, $\mathrm{X} / \mathrm{D}=3.8, \mathrm{Rey}=2.52 \times 10^{5}$
Unfortunately, the important comparison of Pitot pressure profiles near the inlet exit plane cannot lead to a definitive quantification. The basic issue is a possible time-dependent or unrepeatable flow behavior at the inlet exit plane. In Figure 11, two sets of data taken at different dates are presented together with computing results at a streamwise location of $\mathrm{X} / \mathrm{D}=3.8$. The computational simulations with and without DCD actuation are embedded within the data bands. However an important observation is beyond doubt in that the magneto-fluid-dynamic compression has been generated by the surface plasma. From the integrated Pitot pressure data across the exit plane, the increased value produced by the DCD has a range from $8.4 \%$ to $12.6 \%$. The computational simulations yield a value of $6.7 \%$ at the plasma power of 69.0 Watts.

This computed value is lower than the MFD compression gain in Pitot pressure within a constant cross-sectional area rectangular inlet of $11.7 \%$. The two different inlet models are designed for the same cross sectional areas of $9.58 \mathrm{~cm}^{2}$ and the DCD is supplied by similar power (69.0 versus 64.0) for the rectangular inlet. This difference may be attributable to the Mangler effect of boundary-layer thickness or more precisely the reduced displacement thickness over an axisymmetrical configuration.

\section{Features of Computational Simulations}


The basic component of the MFD compression is the magnitude of the electromagnetic perturbation that initiates the viscous interaction. For a DCD it is the intensity of the externally applied electric field through the surface discharge. The electromagnetic perturbation introduces a thermal perturbation to increase the growth rate of the displacement thickness of the boundary layer. The subsequent inviscid-viscous interaction generates a coalescing oblique shock wave for the MFD compression. Since the mechanism is a small perturbation, the compression is generated by a minimum amount of entropy increment. This fact can be verified easily by comparison of the stagnation pressure profiles at the inlet exit. In Figure 12, the stagnation pressure distributions at this location with and without the activated DCD, under identical conditions of the experiments, are depicted

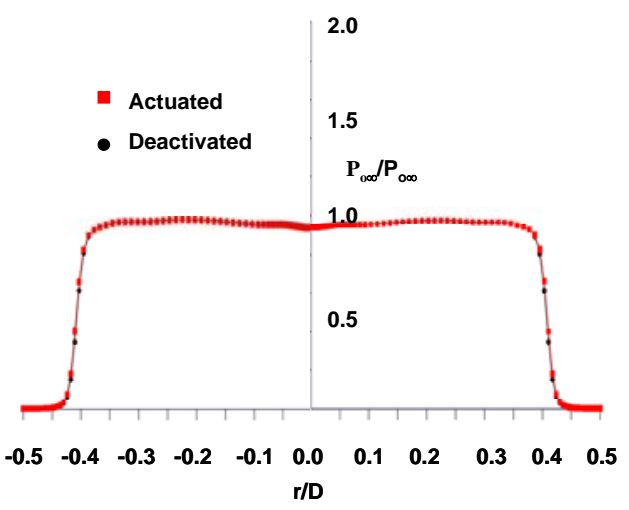

Figure 12. Comparison of stagnation pressure profiles with/without DCD at exit, Rey $=2.52 \times 10^{5}$

together. The DCD is produced with 460 volts and $150 \mathrm{~mA}$. Under this discharging condition the difference in stagnation pressure profiles is negligible which means the additional and small gain in MFD compression occurs without a detrimental effect.

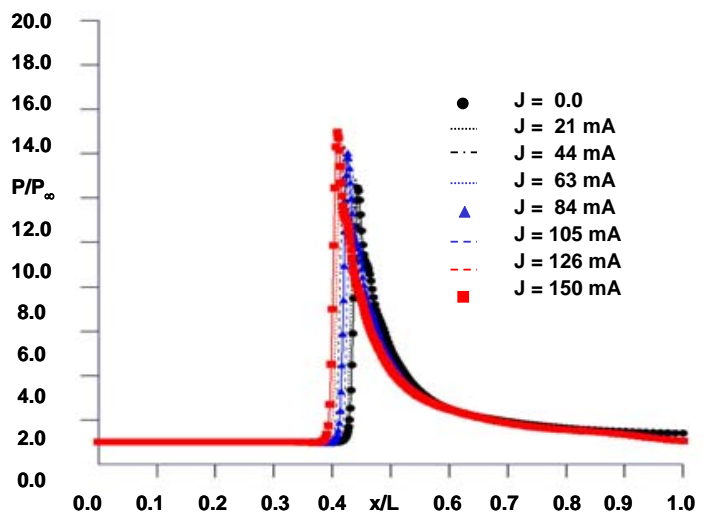

Figure 13. MFD Compression generated by a range of current in external circuit, Rey $=2.52 \times 10^{5}$
The following two graphs, Figures 13 and 14, summarize the application range of a DCD for flow control. In order to examine a large group of DCD conditions, the thermal perturbation is imposed by a simple phenomenological model by matching the Joule heating with a heat source. In practical application, the electric potential is maintained at a constant value and the current flow is modulated by the electrical resistance in the external circuit.

In Figure 13, eight static pressure distributions along the axis of the cylindrical inlet are presented for external circuit current from 0.0 to $150 \mathrm{~mA}$. The maximum discharge current is determined from the experimental observation beyond which a diffusive discharge becomes constricted. The resultant oblique shock by MFD compression increases its strength and the shock angle becomes steeper to intercept the axis of symmetry of the cylindrical inlet at an upstream location. A rapid expansion ensues downstream and the greater the compression the more rapid the expansion occurs. Therefore, an optimal placement of the cathode can generate a desired compression at a specific location of the inlet.

The corresponding static pressure profiles at the inlet exit plane are given in Figure 14. The change in the profile for different discharge currents is rather limited, thus only the current of $0.0,84$, and $150 \mathrm{~mA}$ are highlighted. The main feature of the increased compression is concentrated near the shock front and the downstream expansion is proportional to this strength. In fact, the expanded pressure level at the axis of the inlet by the higher discharge current is actually below the unperturbed condition. This expansion within the inlet can be adjusted by the placement of the electrode closer to the inlet exit to yield a higher compression gain at the inlet exit. However, the induced pressure plateau by the pressure interaction will be lower because the interaction

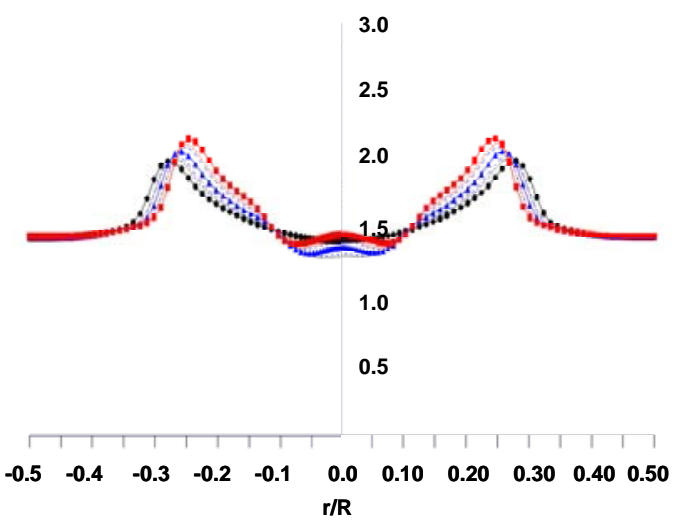

Figure 14. The static pressure profiles at exit for a range of discharge currents, Rey $=2.52 \times 10^{5}$ 
parameter $\chi$, is inversely proportional to the Reynolds number based on the running length from the leading edge. This observation suggests an optimal electrode placement has not been used in the studied configuration.

\section{Concluding Remarks}

The concept of using an electromagnetic perturbation to enhance the pressure interaction for MFD inlet compression has been further demonstrated. The range of application for the high-speed plasma actuator has been extended to include a cylindrical inlet. The power input for plasma generation in the present investigation is limited to 69.0 Watts $(\varphi=460 \mathrm{~V}$ and I $=150 \mathrm{~mA}$ ); therefore the effect of magneto-aerodynamic compression is small. The induced magneto-fluid-dynamic compression is determined to be $6.7 \%$ over that of the unperturbed inlet flow. A reasonable agreement is achieved between experimental and computational results, but a crucial quantification by comparing the measured Pitot pressure profiles near the constant cross-sectional area cylindrical inlet is not achieved, because of the rather large data scattering. An effort shall be sustained to better understand the underlying physics.

The effectiveness of the MFD compression is expected to be less than that of a rectangular inlet partially due to the Mangler effect, but the induced MFD compression is still perceptible and detected by both the experimental and computational investigations. The compression ratio is rather modest at a low power supply and the relatively low free-stream Mach number. Under this circumstance, the MFD compression gain is achieved without any loss of the stagnation pressure by the virtual variable inlet cowl.

\section{Acknowledgment}

The sponsorship by Dr. John Schmisseur and Dr. Fariba Fahroo of AFOSR is deeply appreciated. Fruitful and stimulating discussions with Dr. Donald Paul, Dr. Alan Garscadden, Dr. Datta Gaitonde, and Dr. Roger Kimmel of AFRL, Dr. James Menart of Wright State University, as well as Dr. Sergey Surzhikov of the Russian Academy of Science are sincerely acknowledged.

\section{Reference}

1. Murthy, S.N.B. and Curran, E.T., High Speed Flight Propulsion Systems, Progress in Aeronautics and Astronautics, Vol. 137, AIAA, 1991.

2. Kurth, G., Critical Physical Phenomena in Scramjet Propulsion, AGARD-CP-600, 2002, pp. C5.1- C5.11.

3. Bityurin, V., Kilmov, A., Leonov, S., Lutsky, A, Van Wie D., Brovkin, V., and Kolesnichenko, Yu., Effect of Hetrogenous Discharge Plasma on Shock Wave Structure and Propagation, AIAA 99-4940, Norfolk VA, Nov.

4. Leonov, S.B., Yarantsev, D.A., Gromov, V.G., and Kuriachy, A.P., Mechanisms of Flow control by nearsurface Electrical Discharge Generation, AIAA 2005-0780, Reno NV, January 2005.

5. Shneider, M.N. and Macheret, S.O., Hypersonic Aerodynamic Control and Thrust Vectoring by Nonequilibrium Cold-Air MHD Devices, AIAA 2005-0979, Reno NV, January.

6. Leonov, S., Bityurin, V., Savelkin, K., and Yarantsev, D., Effect of Electrical Discharge on Separation Processes and Shock Position in Supersonic Airflow, AIAA 2002-0355. Reno NV, January 2002.

7. Bityurin, V.A., Bocharov, A.N., and Lineberry, J.T., Results of Experiments on MHD Hypersonic Flow Control, AIAA 2004-2263, Portland OR, June 2004.

8. Shang, J.S. and Surzhikov, S.T., Magnetoaerodynamic Actuator for Hypersonic Flow Control, AIAA Journal Vol. 43, No. 8, August 2005, pp. 1633-1643.

9. Shang, J.S. Surzhikov, S.T., Kimmel, R. Gaitonde, D.V., Hayes, J.R., and Menart, J., Mechanisms of Plasma Actuators for Hypersonic Flow Control, Progress in Aerospace Sciences, Vol. 41, No. 8, Nov. 2005, pp.642-668.

10. Updike, G., Shang, J.S., and Gaitonde, D.V., Hypersonic Separated Flow Control Using MagnetoAerodynamic Interaction, AIAA 2005-0164, Reno NV, January 2005.

11. Poggie, J., Computational Studies of High-Speed Flow Control with Weakly Ionized Plasma, AIAA 20050784, Reno NV, January 2005.

12. Gaitonde, D.V., Simulation of Local and Global High-Speed Flow Control with Magnetic Field, AIAA 2005-0560, Reno NV, January 2005.

13. Borghi, C., Carraro, M., and Cristofolini, A., An Axi-Symmetric Hall Configuration for the MHD Interaction in Hypersonic Flows, AIAA 2005-4785, Toronto Canada, June 2005. 
14. Surzhikov, S.T., and Shang, J.S., The Hypersonic Quasineutral Gas Discharge Plasma in a Magnetic Field, Proceedings third MIT Conference on Computational Fluid and Solid Mechanics, June 14-17, 2005, pp1004-1005.

15. Raizer, Yu. P., Gas Discharge Physics, Springer-Verlag, Berlin, 1991.

16. Mitchner, M. and Kruger, C.H. Jr. Partially Ionized Gases, John Wiley \& Sons, 1973, pp.188-198.

17. Sutton, G.W. and Sherman, A., Engineering Magnetohydrodynamics, McGraw-Hill, NY, 1965, pp.295308.

18. Shang, J.S., Electromagnetic Perturbation to Hypersonic Viscous-Inviscid Interactions, AIAA 2006-0709, Reno NV, January 2006.

19. Shang, J.S., Menart, J., Kimmel, R., and Hayes, J., Magneto-Fluid-Dynamics Hypersonic Inlet with Plasma induced Compression, AIAA 2006-0764, Reno NV, January 2006.

20. Shang, J.S. Menart, J., Kimmel, R., and Hayes, J., Experimental Investigation of Magneto-Fluid-Dynamic Compression in a cylindrical inlet, AIAA 2007-0399, Reno NV, January 2007.

21. Surzhikov, S.T. and Shang, J.S. Two-Component Plasma Model for Two-Dimensional Glow Discharge in Magnetic Field, J. Computational Physics, Vol. 199, 2, Sept. 2004, pp. 437-464.

22. Hayes, W.D., and Probstein, R.F., Hypersonic Flow theory, Academy Press, 1959.

23. Rumsey, C., Biedron, R., and Thomas, J., CFL3D: Its History and Some Recent Applications, NASA TM112861, May 1007.

24. Thomas, J.L., Diskin, B., Brandt, A., Textbook Multigrid Efficiency for Fluid Simulation, Annual Review of Fluid Mechanics, Vol. 35, January 2003, pp.317-340.

25. Hornung, H., Regular and Mach Reflection of Shock waves, Ann. Rev. Fluid Mech., Vol. 18, 1986, pp.3358.

26. M. S. Ivanov, G. N. Markelov, A. N. Kudryavtsev, and S. F. Gimelshein, Transition between Regular and Mach Reflection of Shock Waves in Steady flow, AIAA-1997-2511, June 1997. 